\title{
Bildung eines insulinartigen Wirkstoffes durch die isolierte Rattenleber
}

\author{
Von \\ E. Siess, A. Teinzer, E. Strudck und O. Wieland
}

Aus dem Laboratorium für klinische Chemie und Biochemie (Leiter: Prof. Dr. O. WieIAND) der II. Medizinischen
Universitätsklinik, München (Direktor: Prof. Dr. G. Bodechted)

Eingegangen am 10. März 1965

Summary. Perfusion of isolated rat liver for periods up to six hours resulted in the steady release of a substance - of hepatic origin - which stimulates the oxydation of glucose-1 ${ }^{14} \mathrm{C}$ to ${ }^{14} \mathrm{CO}_{2}$ by rat epididymal adipose tissue. The activity of the substance, therefore, resembles that of insulin ("hepatic ILA"); however, the activity is not suppressed by the addition of antiinsulin antibodies obtained in guinea pigs. This hepatic IL $A$ is released whether or not pancreatic insulin is added to the circulating medium and thus does not represent a metabolite of added pancreatic insulin. It has properties similar to those of "non-suppressible" ILA of human serum. The rate of production of hepatic ILA in the perfused rat liver is sufficient to warrant consideration of its contributing to a major extent to the maintenance of serum levels of "non-suppressible" ILA, particularly in pancreatectomized animals. Also described in this paper is an activation of the biologic activity of crystalline in sulin as a result of heating in bicarbonate buffer (Table 1 ) and it is suggested on the basis of ultracentrifugation studies that this might be the result of "disaggregation" of insulin into smaller units.

Résumé. Au cours de perfusions de foie isolé de rat, les auteurs décrivent, pendant des périodes allant jusqu' à six heures, la formation d'une substance hépatogène capable de stimuler l'oxidation de glucose $-1-{ }^{14} \mathrm{C}$ en ${ }^{14} \mathrm{CO}_{2}$ par la graisse épididymaire de rat. Cette substance a donc une activité de type insulinique ("ILA hépatique»), mais cette activité n'est pas supprimée par l'adjonction d'anticorps anti-insuline obtenus chez le cobaye. La formation de cette ILA hépatique ne nécessite pas la présence d'insuline pancréatique exogène dans le milieu de perfusion et n'est done pas l'insuline pancréatique exogène modifiée. Son activité ressemble à celle de l' "IL A non supprimable» du sérum humain. La production de cette substance par le foie perfusé est suffisamment active pour qu'il soit possible d'admettre qu'elle contribue peut-être à la formation de l'activité insulinique nonsupprimable du sémum et done à sa persistance après pancréatectomie. Le rapport faitégalement état (Tableau 2) d'une activation thermique de l'effet biologique de solution d'insuline dans un tampon bicarbonate.

Zusammenfassung. Die isolierte Rattenleber gibt bei Durchströmung bis zu 6 Stunden eine Substanz an das Medium ab (,hepatische ILA“), die wie Insulin die Glukose-1- ${ }^{14} \mathrm{C}$-Oxydation durch das Rattennebenhodenfettgewebe stimuliert, jedoch durch Insulinantikörper aus Meerschweinchenserum nicht unterdrückbar ist. Die hepatische ILA ist nicht an die Zufuhr von Pankreasinsulin gebunden, stellt also kein Umwandlungsprodukt von freiem Insulin dar. Sie ähnelt in ihren Eigenschaften der nichthemambaren Serum-ILA des Menschen. Entsprechend der Bildungsrate hepatischer ILA im Perfusionsversuch könnte die Leber quantitativ einen maßgeblichen Beitrag zur Aufrechterhaltung der nichthemmbaren SerumILA liefern. Im Zuge der vorliegenden Untersuchungen wurde außerdem beobachtet, daß die biologische Aktivität von Rinder-Kristallinsulin durch kurzes Erwärmen im Bicarbonatpuffer zunimmt (Tabelle 1). Der Effekt beruht möglicherweise auf einer thermischen Dissoziation des Insulins in Untereinheiten.
1959 berichtete LEONaRDs über das Vorkommen zweier Substanzen im Blutserum mit insulinartiger Wirkung auf die in vitro ${ }^{14} \mathrm{CO}_{2}$-Bildung aus $1-{ }^{14} \mathrm{C}$ markierter Glukose durch das epididymale Fettgewebe der Ratte ${ }^{30}$. Nach diesen Befunden war nur ein kleiner Teil der Gesamtinsulinaktivität (ILA) des Serums mit Insulinantikörpern (IAK) hemmbar. Spätere Untersucher sprachen von einem ,freien" "4, ,hemmbaren“17, ,typical"44, 44, ,circulating“"5 bzw. von dem ,gebundenen“, „,nichthemmbaren“, ,atypical " oder, ,pancreatic" Insulin, um das Pankreasinsulin, dessen Primärstruktur für viele Tierarten aufgeklärt wurde $38,3,25,56,22$, von dem noch weitgehend unbekannten Stoff mit insulinähnlicher Wirkung (ILA) zu unterscheiden. Ganztierversuche mit dem Ziel, die Quelle der nichthemmbaren ILA aufzufinden, führten SamaAN et al. zu der Ansicht, daß die Hundeleber in der Lage sei, ,typical " TLA in die atypische Form überzuführen $^{43}$. BÜRGI et al. versuchten daraufhin nach Angebot von hemmbarem Ratteninsulin an die isolierte
Rattenleber den direkten Nachweis der Umwandlung in nichthemmbare ILA zu führen, was jedoch - selbst unter Anwendung von Insulinmengen bis zu $10^{4} \mu \mathrm{E} / \mathrm{ml}$ Perfusat nicht gelang ${ }^{5}$. Nach eigenen Untersuchungen, über die im folgenden berichtet wird, scheint dagegen der Leber eine quantitativ beachtliche Rolle bei der Bildung einer nicht durch IAK hemmbaren ILA (,hepatische" ILA) zuzukommen. Über einige dieser Ergebnisse ist von E. SIEss schon kurz an anderer Stelle berichtet worden ${ }^{47}$.

\section{Methodik}

Antikörpererzeugung: Die Methode der AK-Erzeugung beim Meerschweinchen nach MoronEY et al. ${ }^{37}$ durch Injektion eines gleichteiligen Gemisches aus FREUNDS Adjuvans ${ }^{15}$ und Rinderkristallinsulin in $0,004 \mathrm{~N} \mathrm{HCl}$ in 14-tägigen Abständen wurde abgeändert, um die auch von anderer Seite ${ }^{55}$ beobachteten, an der Injektionsstelle auftretenden Geschwürsbildungen 
zu vermeiden. Dies gelang bei Anwendung eines Antigengemisches aus 2 Teilen Insulinlösung* $(2 \mathrm{mg} / \mathrm{ml}$ ) in $0,004 \mathrm{~N} \mathrm{HCl}, 1$ Teil Tween 80 and 1 Teil Paraffinöl, das unter die Nacken- oder die Rückenhaut injiziert wurde. $1 \mathrm{ml}$ dieser Mischung wurde pro Tier in 14-tägigen Abständen verabreicht. In ihrer Wirksamkeit standen die auf diese Weise erzeugten AK den mit FREUNDS Adjuvans erhaltenen nicht nach.

Gewinnung der Antiseren: Die Blutentnahme erfolgte am 10. Tag nach der letzten Injektion (nach 6- bis 7maliger Immunisierung) durch Herzpunktion in Äthernarkose. Das Blut wurde 1 bis 2 Std bei Raumtemperatur und anschließend über nacht bei $4^{\circ} \mathrm{C}$ stehen gelassen und am nächsten Tag zentrifugiert. Die meisten Seren waren leicht hämolytisch. Sie wurden auf $50 \mathrm{ml}-$ Portionen verteilt und in der Tiefkühltruhe bei $-20^{\circ} \mathrm{C}$ aufbewahrt.

Antikörperanreicherung: Zur AK-Isolierung trennten wir zunächst nach HoŘsší et al. ${ }^{24}$ - modifiziert nach LI et al. ${ }^{31}$ - die Gammaglobuline durch Rivanolfällung ab: $\mathrm{Zu} 25 \mathrm{ml}$ Serum (pH 8,5) ließen wir unter

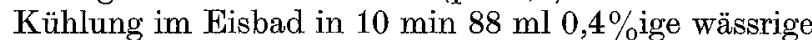
Rivanollösung ( $\mathrm{pH}$ 8,5) unter mechanischem Rühren zutropfen. Nach Abzentrifugieren des Niederschlages wurden pro $15 \mathrm{ml}$ Überstand $2 \mathrm{ml}$ gesättigte Ammoniumsulfatlösung zugegeben. Nach Einstellen des $\mathrm{pH}$ auf 5,5 mit $2 \mathrm{~N} \mathrm{H}_{2} \mathrm{SO}_{4}$ wurde die klare Lösung zur $\mathrm{Ab}$ trennung des Rivanols über eine Amberlite IRC - 50 Säule (innerer Durchmesser $4 \mathrm{~cm}$, Höhe des Austauscherharzes $2.5 \mathrm{~cm}$ ) geschickt, die mit $0,2 \mathrm{M}$ Kaliumphosphatpuffer $\mathrm{pH} 5,5$ - enthaltend $0,5 \mathrm{M}$ Ammoniumsulfat - vorgewaschen war. Die AK-haltigen Eluate wurden 8 bis 10 Std gegen dest. Wasser bei $4^{\circ} \mathrm{C}$ dialysiert und dann gefriergetrocknet. Die Lyophilisate lösten wir in $0,005 \mathrm{M} \mathrm{K}_{2} \mathrm{HPO}_{4}$ und dialysierten nochmals bis zur Einstellung konstanter Leitfähigkeit und $\mathrm{H}^{+}$-Konzentration gegen die gleiche Phosphatlösung.

Zur weiteren Reinigung wurde die Proteinlösung, die im wesentlichen nur noch $\gamma$-Globulin enthielt, auf eine DEAE-Cellulosesäule (innerer Durchmesser $2 \mathrm{~cm}$, Höhe des Austauschermaterials $35 \mathrm{~cm}$ ) aufgetragen und mit steigenden Phosphatkonzentrationen beginnend von $0,005 \mathrm{M} \mathrm{K}_{2} \mathrm{HPO}_{4}$ bis $0,12 \mathrm{M} \mathrm{KH}_{2} \mathrm{PO}_{4}$ und fallendem $\mathrm{pH}$-Gradienten nach SoBER et al. ${ }^{52}$ in der Modifikation von FAHEY et al. ${ }^{14}$ eluiert. Nach diesem Vorgehen fand sich die AK-Aktivität auf die Fraktionen 9 bis 16 (je $5,5 \mathrm{ml}$ ) verteilt. Sie wurden vereinigt und bei $4^{\circ} \mathrm{C}$ aufbewahrt. Hierbei blieb die Aktivität mindestens 3 Wochen unverändert. Dagegen trat durch wiederholtes Einfrieren und Auftauen rascher Wirksamkeitsverlust ein.

Bei einer Ausbeute von 50-80\% stieg nach dem beschriebenen Anreicherungsverfahren die spez. AKAktivität um das 20- bis 30-fache gegenüber Nativserum an. Die Vorreinigung mit Rivanol scheint wesentlich zu sein, da andere Autoren durch direkte säulenchroma-

* Für die Úberlassung von Rinder-Kristallinsulin danken wir Herrn Prof. H. MAskE, Farbwerke Hoechst A. G., Frankfurt-Hoechst. tographische Auftrennung des Antiserums nur 10-fache Anreicherung erzielten ${ }^{60}$. In der Ultrazentrifuge sedimentierte unsere Fraktion als symmetrischer Gipfel mit einer Sedimentationskonstante von 6,58 Svedberg-E.*

Titerbestimmung: Die Bestimmung des AK-Titers führten wir im biologischen Test mit dem Nebenhodenfettgewebe der Ratte durch. Die von uns vereinfachte Methode der Gewebeinkubation und der Messung des aus $1-{ }^{14} \mathrm{C}$ markierter Glukose entwickelten markierten $\mathrm{CO}_{2}$ wird an anderer Stelle ausführlich beschrieben $^{48}$.

Zur Bestimmung der AK-Aktivität wurden jeweils $0,2 \mathrm{ml}$ Insulinlösung mit einem Gehalt von $4000 \mu \mathrm{E}$ Rinderinsulin mit $0,2 \mathrm{ml}$ AK-Lösung gemischt, 90 min bei $37^{\circ} \mathrm{C}$ inkubiert und von der Mischung $0,05 \mathrm{ml}$ in den Fettgewebstest eingesetzt. Zur Standardisierung des Titers wurde diejenige AK-Verdünnung gewählt, die Halbhemmung einer Eichdosis von $500 \mu \mathrm{E}$ Insulin pro Ansatz bewirkte. Um diese AK-Konzentration zu ermitteln, mußten für jede Titerbestimmung mindestens vier verschiedene Verdünnungen getestet werden. Aus der hierbei erhaltenen Hemmungskurve wurde der Titer interpoliert. Da zwischen AK-Verdünnung und der nach der Vierpunkt-Eichkurve noch vorliegenden Insulinaktivität eine nichtlineare Beziehung gefunden wurde ${ }^{46,}$ muß man sich zur Titerbestimmung in einem Bereich bewegen, der nicht zu weit von der Halbhemmungskonzentration abweicht.

Bestimmung der hemmbaren- und nichthemmbaren ILA im Perfusionsmedium: Die der Mischkammer entnommenen Perfusatproben $(8 \mathrm{ml}$ ) wurden bis zur Beendigung der Durchströmung bei $4^{\circ} \mathrm{C}$ stehen gelassen, sodann gemeinsam bei Zimmertemperatur 5 min zentrifugiert und die Überstände über nacht bei $4^{\circ} \mathrm{C}$ gegen phosphatfreie Krebs-Bicarbonat-Ringerlösung $\left(\mathrm{KBR},{ }^{27}\right)$ dialysiert. Im Test wurden zu je $1 \mathrm{ml}$ Dialysat $0,1 \mathrm{ml}$ gereinigter AK-Lösung (entsprechend ca. $100 \mu \mathrm{g}$ Protein) oder anstatt dessen $0,1 \mathrm{ml}$ KBR gegeben. Nach 90 min Inkubation der Ansätze bei $37^{\circ} \mathrm{C}$ wurden aliquote Mengen von je 0,4 $\mathrm{ml}$ für die ILA-Bestimmung eingesetzt.

Leberperfusion: Die Durchströmung der isolierten Rattenleber erfolgte im rezirkulierenden System nach MrLler et al. ${ }^{35}$ und nach Brauter et al. ${ }^{2}$. Die Einzelheiten der Methode und die Zusammensetzung des Perfusionsmediums nach ${ }^{45}$ sind an anderer Stelle ausführlich beschrieben ${ }^{34}$. Als Spendertiere dienten männliche Sprague-Dawley Ratten mit einem Körpergewicht von $200-250 \mathrm{~g}$ (Lieferant: W. Gassner, MünchenOttobrunn), die mit einer Standard-Diät (Altromin $\mathbf{R}$, Fa. Lage/Lippe) und Wasser ad libitum gefüttert warden.

Um die Anoxiephase zu verkürzen, wurde die Präparation der Leber folgendermaßen abgeändert: Vor Kanülierung der Pfortader wurde eine feste Ligatur an der

* Für dje Ultrazentrifugen-Untersuchungen sind wir Fräulein I. HöFER, Spinco-Abt. der Fa. Beckman Instr., München, zu großem Dank verpllichtet. 
Stelle der Vereinigung der Mesenterialvenen gelegt. Der Faden, mit dem die Pfortader im lebernahen Bereich unterlegt war, wurde unter leichtem Zug gehalten, so daß für den Augenblick des Einführens der Kanüle Blutleere im Operationsgebiet der V.portae herrschte. Dies erleich terte den Vorgang wesentlich. An die liegende Pfortaderkanüle wurde unmittelbar eine Infusion von oxygeniertem Blutgemisch (ohne Antibioticumzusatz) in situ angeschlossen, wodurch die Anoxiephase auf $30-60$ sec abgekürzt wurde. Nach der Kanülierung der V.cava sup. in üblicher Weise wurde der Oberkörper der Ratte oberhalb des Zwerchfells abgetrennt, so daß die Durchströmungsflüssigkeit ungehindert durch die nun freiliegende Kanüle der oberen Hohlvene abfließen konnte. Zuletzt wurde die Leber herauspräpariert und an die Perfusionsapparatur angeschlossen, was eine nochmalige kurze Anoxieperiode von maximal 5 sec verursachte. Vor Beginn der eigentlichen Versuche wurde die Perfusion zur Einstellung konstanter Bedingungen 30-45 min laufen gelassen.

Da damit zu rechnen war, daß Zink, welches nach MASk $E^{32,33}$ zusammen mit Insulin das Pankreas verläßt, möglicherweise in den weiteren Stoff wechsel des Insulins eingeschaltet ist, stellten wir in Nachahmung der physiologischen Verhältnisse durch Zugabe von $0,5 \mathrm{ml} \mathrm{ZnSO}_{4}$-Lösung das Perfusionsmedium auf einen Zinkspiegel von $1 \mu \mathrm{g}$ pro $\mathrm{ml}$ ein ${ }^{13 a}$. $10-15$ min später erfolgte die erste Blutentnahme, im folgenden als Nullabnahme bezeichnet. Weitere Proben wurden jeweils in einer Menge von $8 \mathrm{ml}$ nach $30,60,90,120,180$, 240,300 and $360 \mathrm{~min}$ abgenommen. In den Durchströmungsversuchen mit Insulin wurden in der Regel $20 \mathrm{mE}$ Rinderkristallinsulin (Farbwerke Hoechst) in die Mischkammer gegeben und sofort anschließend mit einer automatischen Infusionsspritze $18 \mathrm{mE}$ pro Std laufend bis zum Beginn der fünften Std zugeführt.

\section{Ergebnisse}

Abb. 1 zeigt das Ergebnis eines Versuches, in dem die Leber mit Zusatz von Insulin durchströmt wurde. Die Gesamtinsulinaktivität sowie die durch $\mathrm{AK}$ nicht. hemmbare ILA des Perfusates - ausgedrückt in Nettoimpulsen ${ }^{14} \mathrm{CO}_{2}$ - sind in Abhängigkeit von der Perfusionsdauer aufgetragen. Zur Berechnung der Netto- ${ }^{14} \mathrm{CO}_{2}$-Produktion wurde die ${ }^{14} \mathrm{CO}_{2}$-Bildung des Fettgewebes in reiner Pufferlösung von der Gesamtzahl der gemessenen Tmpulse in Abzug gebracht.

Bei großem AK-Überschuß beobachteten wir regelmäBig weniger Impulse, als der Gewebeeigenproduktion entsprach. Dieses Verhalten dürfte auf die Neutralisation endogenen, am Fettgewebe haftenden Insulins zurückzu. führen sein. Dies ließ sich durch Untersuchungen der Insulinbindung am Fettgewebe erhärten: Inkubiert man Fettgewebe bei $25^{\circ} \mathrm{C}$ in $0,5 \mathrm{ml} \mathrm{KBR}$ mit $1000 \mu \mathrm{E}$ Kristallinsulin verschieden lange Zeiton und testet anschließend. in einem insulinfreien KBR-Medium, so findet man das Maximum der ${ }^{14} \mathrm{CO}_{2}$-Bildung (und damit der Insulinfixierung) nach etwa $10 \mathrm{~min}$. Inkubation. Um die Festigkeit der Gewebehaftung zu prüfen, wurden etwa $20 \mathrm{mg}$ schwere Fettgewebstückchen wie beschrieben 20 min inkubiert und sodann durch leichtes Hin- und Herschwenken in insulinfreiem Medium gewaschen. Nach 20 min Waschdauer verliert das Fettgewebe durch Fortsetzung der Wäsche nur mehr sehr wenig an Aktivität. In den Waschflüssigkeiten stiegen die Impulse korrespondierend an. Die Erscheinung der Insulinbindung am Fettgewebe, jedoch un. ter Einsatz 500mal höherer Insulinkonzentrationen, wurde schon von HAUGAARD et al. beobachtet ${ }^{23}$.

Aus Abb. 1 geht hervor, daß die nichthemmbare ILA im Durchströmungsmedium kontinuierlich über

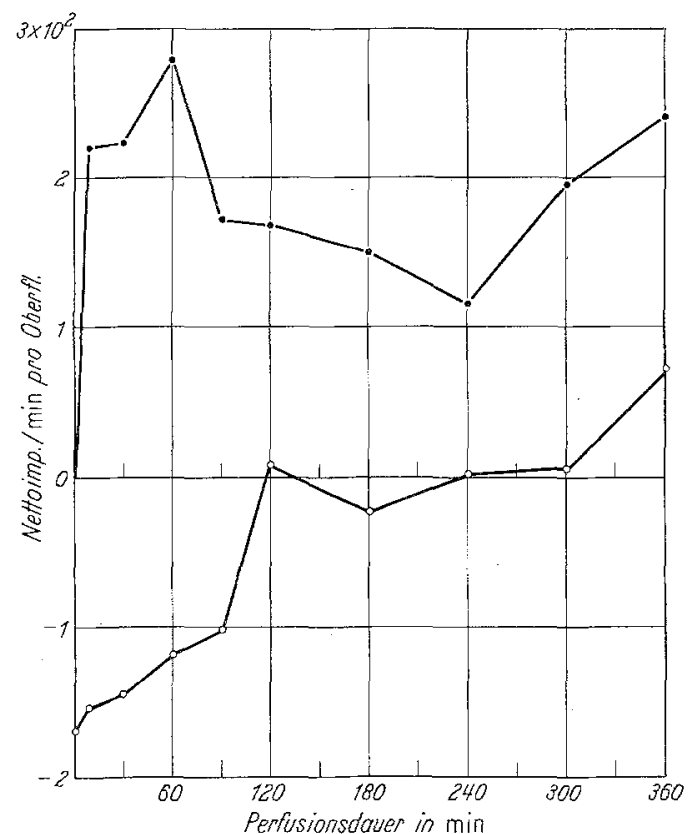

Abb. 1. Verhalten der Gesamt-TLA und der nichthemmbaren ILA im Verlauf einer Jeberperfusion. Die ILA-Alstivitäten sind in ${ }^{14} \mathrm{CO}_{2}$-Nettoimpulsen pro min - bezogen auf Fettgewebso berfläche ${ }^{48}$ - angegeben. Zum Zeitpunkt 0 wurden $20 \mathrm{mE}$ krist. Rinderinsulin als Stoß und anschließend $18 \mathrm{mE} / \mathrm{Std}$ als Dauerinfusion über 5 Std. zugegeben. Vol. $150 \mathrm{ml}$. - Gesamt-ILA O-O nichthèmmbare ILA

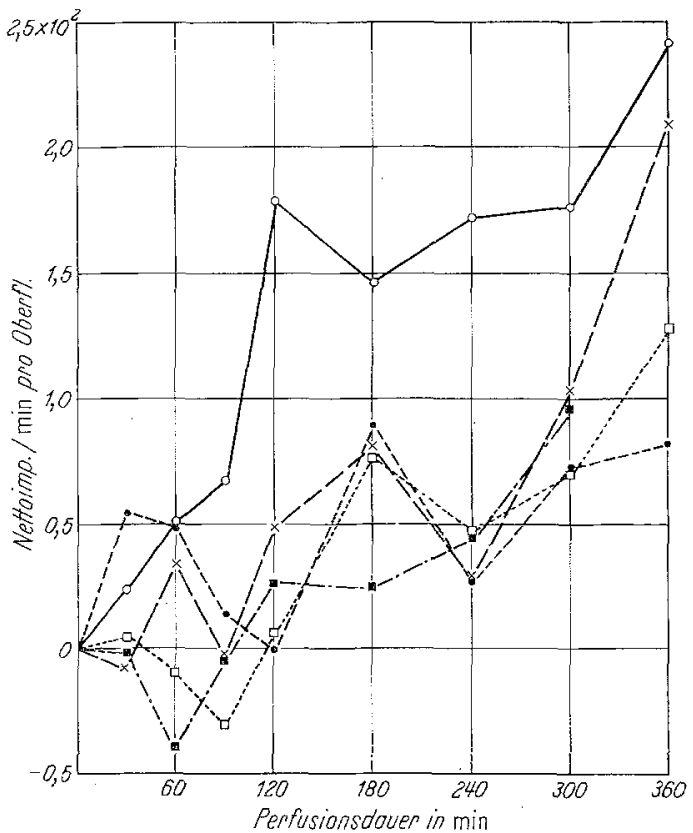

Abb. 2. Bildung nichthemmbarer ILA durch die isolierte Rattenleber. Aktivitätsangabe wie in Abb. 1. Gegenüberstellung der Experimente mit und ohne Insulinzusatz zum Hedium

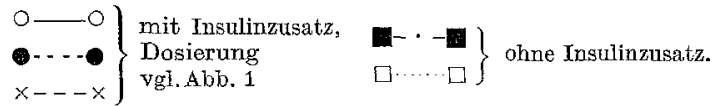


die gesamte Versuchsdauer von 6 Std ansteigt. Daß es sich hierbei nicht um eine Umwandlung des zugesetzten Insulins in eine nichthemmbare Form handelt, ergab sich in Experimenten, in denen die Leber mit insulinfreiem Medium perfundiert wurde. In Abb. 2 erkennt man, daß es auch in Abwesenheit von Insulin zu einer ähnlichen Bildung nichthemmbarer ILA kommt, wie in den Vergleichsversuchen mit Insulin.

Um Aufschluß über die Menge der gebildeten hepatischen ILA zu erhalten, wurde in vier weiteren Experimenten die Gesamtzahl der nach fünfstündiger Perfusion vorliegenden $\mu \mathrm{E}$ bestimmt. Es fanden sich in einem Fall 100 (nach 3 Std 77), in den anderen Fällen 60,45 und $55 \mu \mathbf{E}$ nichthemmbarer ILA pro $\mathrm{ml}$. Dies entspricht einer mittleren Kapazität der Rattenleber zur Bildung von $1300 \mu \mathrm{E} / \mathrm{Std}$.

Wie zu erwarten, tritt bei „Leerperfusion“, d.h. im analogen System ohne Leber, keine nichthemmbare ILA auf (Abb. 3).

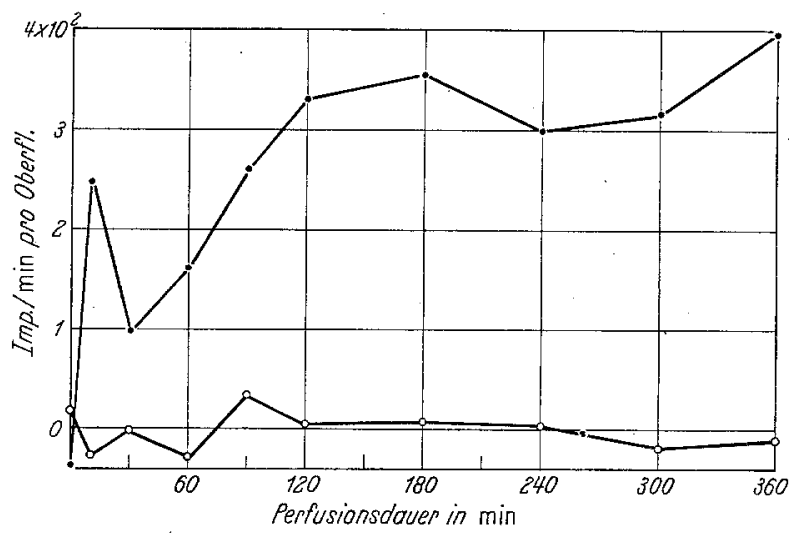

Abb. 3. Kontrollperfusion ohne Leber. Insulinaktivität des zirkulierenden Mediums mit (O__-O) und ohne (C-O) Zusatz von Insulinantikörper. Zum Zeitpunkt 0 einmalige Zugabe von $40 \mathrm{mE}$ Rinderinsulin. Durchströmungsvolumen $150 \mathrm{ml}$

Bei diesem Versuch ergab sich als bemerkenswerter Nebenbefund, daß die Aktivität des zugesetzten Insulins im Laufe der Durchströmung anstieg, obwohl nur eine einmalige anfängliche Zugabe erfolgt war. Dies steht möglicherweise mit folgender Beobachtung im Zusammenhang, wonach Insulin durch Erwärmen aktiviert wird. So führt z. B. 10 min Inkubation des verwendeten Rinderinsulins in $\mathrm{KBR}$ bei $50^{\circ} \mathrm{C}$ zu einer 8 - 10 fachen Aktivitätssteigerung. Diese Versuche sind in Tab. 1 zusammengestellt. Aus Untersuchungen mit der Ultrazentrifuge* ergaben sich Hinweise dafür, daß die Erwärmung zu einer Disaggregation des Insulins in kleinere, biologisch aktive Untereinheiten führt. Der Aktivitätssteigerung des zugesetzten Insulins während der 7 -stündigen Blindperfusion bei $37^{\circ} \mathrm{C}$ (vgl. Abb. 3) könnte eine ähnliche thermische Beeinflussung zugrunde liegen.

Zur näheren Charakterisierung des von der Leber gebildeten Faktors wurden einige seiner Eigenschaften im Vergleich zu Kristallinsulin und zu nichthemmbarer menschlicher Serum. ILA untersucht. Wie Tab. 2

* vgl. Fußnote S. 22.
Tabelle 1. Thermische Aktivierung von Rinder-Kristallinsulin. Insulinlösungen von $100 \mu g / m l ~ K B R$ wurden $10 \mathrm{~min}$ bei den angegebenen Temperaturen inkubiert und anschliessend mit der Fettgewebsmethode getestet. Vor dem Test wurde zur Korrektur der pH-Verschiebung 2,5 min mit Carbogen begast

\begin{tabular}{ccc}
\hline Versuch & $\begin{array}{c}10 \begin{array}{c}\text { min inknbiert } \\
\text { bei }{ }^{\circ} \mathrm{C}\end{array} \\
\text { naktivität }\end{array}$ & $\begin{array}{c}\text { Insulin- erwärmt } \\
\text { akcht erwärmt }\end{array}$ \\
\hline 1 & 70 & 3,5 \\
2 & 70 & 4,0 \\
3 & 70 & 3,5 \\
4 & 50 & 9,6 \\
5 & 50 & 7,9
\end{tabular}

Tabelle 2. Eigenschaften der nichthemmbaren ILA der Rattenleber im Vergleich zu Kristallinsulin und zu nichthemmbarer. Serum-ILA. Lit. vgl. (41,21)

\begin{tabular}{cccc}
\hline & Kristall- & Serum- \\
insulin & ILA & hepatische \\
\hline
\end{tabular}

Hemmbar durch Insulin-

Antikörper

Inaktivierbar durch Cystein

Inaktivierbar durch Wärme $\left(50^{\circ} \mathrm{C}, 10 \mathrm{~min}\right)$

Dialysierbar

Extrahierbar mit HCl-Alkohol

$\begin{array}{lll}+ & + & - \\ + & \frac{-}{+} & ++ \\ + & + & -\end{array}$

zeigt, weist unsere Substanz Ähnlichkeiten mit der nichthemmbaren Serum-ILA auf, deren von FroEscH et al. beschriebene Merkmale ${ }^{16,17}$ wir bestätigt fanden. Bei Wärmebehandlung erwies sich die hepatische ILA allerdings als beträchtlich labiler. Bei Extraktion mit HCl-Äthanol betrug die Ausbeute etwa 50\%.

\section{Diskussion}

Nach den vorliegenden Untersuchungen könnte der Leber eine maßgebliche Rolle als Produktionsstätte nichthemmbarer Serum-ILA zukommen. Nach BÜRGI et al. beträgt der Serumspiegel der Ratte an nichthemmbarer ILA $110 \mu \mathrm{E} / \mathrm{ml}^{5}$. Bei einer angenommenen Blutmenge der Ratte von $15 \mathrm{ml}$ wäre die Leber bei der von uns gemessenen Kapazität von $1300 \mu \mathrm{E} / \mathrm{Std}$ in der Lage, den gesamten Bestand an nichthemmbarer Serum-ILA in 1-2 Stunden aufzubauen. Hierbei muß allerdings die Frage der Abbaugeschwindigkeit der hepatischen ILA noch offen gelassen werden.

Was den Entstehungsmechanismus der hepatischen IIA betrifft, so ergeben sich aus unseren Versuchen keine Anhaltspunkte für eine Umwandlung freien, mit AK hemmbaren Insulins in eine nichthemmbare Form, wie dies von SamaAn et. al. vermutet wird ${ }^{43}$. Vielmehr ist die ILA-Produktion der isolierten Leber nicht an die Zufuhr von Pankreasinsulin gebunden, sondern stellt eine eigenständige Leistung der Leber dar. BÜBGT et al., die die Frage der Insulinumwandlung durch die Rattenleber ebenfalls in Perfusionsversuchen prüften, konnten nach Zufuhr von Ratteninsulin keine Bildung nichthemmbarer ILA beobachten ${ }^{5}$. Versuche ohne Insulinzusatz wurden von den genannten Autoren nicht durchgeführt. Für die Diskrepanz zwischen ih- 
ren und unseren Ergebnissen, könnten verschiedene methodische Unterschiede verantwortlich sein. Dies gilt vor allem für die Empfindlichkeit der Insulinbestimmungsmethode, deren untere Grenze von der Schweizer Gruppe bei $50 \mu \mathrm{E} / \mathrm{ml}$ gezogen wird, während wir Mengen bis herab zu $20 \mu \mathrm{E} / \mathrm{ml}$ erfaßten. Nach unseren Versuchen betrug die Bildung an hepatischer ILA in 5 Std im Mittel $65 \mu \mathrm{E} / \mathrm{ml}$ Perfusat. Geht man hiervon aus, so wäre es verständlich, daß sich den Schweizer Autoren, die zudem mit einem größeren Perfusionsvolumen $(200 \mathrm{ml})$ arbeiteten, die nichthemmbare ILA dem Nachweis entzog. Ein weiterer Unterschied besteht darin, daß wir dem Perfusionsmedium Zink in physiologischen Konzentrationen zusetzten. Inwieweit Zinkionen tatsächlich einen essentiellen Faktor für die Bildung hepatischer ILA darstellen, und der Achromobacter-Gruppe gefunden*. Wie Kontrollversuche ohne Fettgewebe ergaben, entfallen jedoch auf diese Bakterienverunreinigung maximal $3 \%$ der Gesamtimpulse, so daß auf eine Korrektur verzichtet werden konnte. Auch das infolge Hämolyse zunehmend auftretende Hämoglobin konnte als Störquelle der Insulinbestimmung ausgeschlossen werden ${ }^{46}$.

Bei Leberperfusionen mit Rattenblut fanden CoHer und Gordon eine durchschnittliche Plasmaproteinzunahme von $160 \mathrm{mg}$ in $5 \mathrm{Std}^{7}$. Davon waren etwa $50 \mathrm{mg}$ immunologisch nachweisbares, lebereigenes Protein $^{20}$, während von den restlichen $110 \mathrm{mg}$ rund die Hälfte auf neusynthetisiertes Albumin entfällti9. Da Albumin die Glukoseoxydation des Fettgewebes nicht stimuliert ${ }^{1,26,46}$, wird man die von uns beobachtete hepatische ILA in dem während der Durchströmung

Tabelle 3. Stoffe mit stimulierender Wirkung auf die D-Glukose-1-14C-Oxydation des Fettgewebes. Literaturangaben in Molaritäten wurden in ug umgerechnet

\begin{tabular}{|c|c|c|c|c|c|}
\hline Substanz & $\begin{array}{l}\text { physiol. Blut- } \\
\text { spiegel pro ml }\end{array}$ & Lit. & $\begin{array}{c}\text { in vitro wirk- } \\
\text { same Menge } \\
\text { pro } \mathrm{ml} \\
\end{array}$ & Lit. & $\begin{array}{c}\begin{array}{c}\text { Sobwellendosis } \\
\text { in vitro }\end{array} \\
\text { physiol. } \\
\text { Plasmaspiegel } \\
\end{array}$ \\
\hline ACTH & $5 \times 10^{-3} \mu \mathrm{g}$ & $(10)$ & $10^{2} \mu \mathrm{g}$ & $(28)$ & $2 \times 10^{4}$ \\
\hline Adrenalin & $10^{-7} \mu \mathrm{g}$ & (13) & $2 \times 10^{-1} \mu \mathrm{g}$ & (6) & $2 \times 10^{6}$ \\
\hline Noradrenalin & $10^{-6} \mu \mathrm{g}$ & (13) & $2 \times 10^{-1} \mu \mathrm{g}$ & (6) & $2 \times 10^{5}$ \\
\hline $\begin{array}{l}\text { Glucagon } \\
\text { (reduziert) }\end{array}$ & $3 \times 10^{-1} \mu \mathrm{g}$ & (54) & $4 \times 10^{-1} \mu \mathrm{g}$ & (59) & 1,3 \\
\hline Insulin & $279 \mu \mathrm{E}$ & $(46)$ & $20 \mu \mathrm{E}$ & $(46)$ & $7 \times 10^{-2}$ \\
\hline $\begin{array}{l}\text { ILA nichthemm- } \\
\text { bar, hepatisch }\end{array}$ & $186 \mu \mathrm{E}$ & $\begin{array}{l}\text { (diese } \\
\text { Arbeit) }\end{array}$ & $20 \mu \mathrm{E}$ & $\begin{array}{c}\text { (diese } \\
\text { Arbeit) }\end{array}$ & $1,1 \times 10^{-1}$ \\
\hline Nicotinsäure & $4-8 \mu \mathrm{g}$ & (9) & $123 \mu \mathrm{g}$ & (29) & 20 \\
\hline Oxytocin & $0-80 \mu \mathbf{E}$ & (i2) & $10^{3} \mu \mathrm{E}$ & $(36)$ & 25 \\
\hline Prolactin & $4 \mu \mathrm{g}$ & $(50)$ & $10^{3} \mu \mathrm{g}$ & $(58)$ & 250 \\
\hline STH & $0,2-0,5 \mu \mathrm{g}$ & (11) & $10^{3} \mu \mathrm{g}$ & $(57)$ & $2,5 \times 10^{3}$ \\
\hline Vasopressin & $37 \mu \mathrm{E}$ & (12) & $6 \times 10^{6} \mu \mathrm{E}$ & (39) & $1,6 \times 10^{4}$ \\
\hline
\end{tabular}

bedarf allerdings noch systematischer Untersuchungen. Schließlich könnte es eine Rolle spielen, daß wir zur Entfernung eventueller Hemmstoffe des Insulintestes, wie z.B. Glukosamin ${ }^{49}$, die Perfusatproben routinemäBig dialysierten.

Was die Natur der hepatischen ILA betrifft, so können die bisher bekannten Stoffe mit steigernder Wirkung auf die Oxydation des ersten C-Atoms der Glukose weitgehend ausgeschlossen werden. Die in Frage kommenden Substanzen sind in Tab. 3 zusammengestellt. Aus dem Verhältnis der in vitro wirksamen Mengen zu den physiologisch auftretenden Blutkonzentrationen in der letzten Spalte sieht man, daß nur im Falle des Insulins die physiologischen Konzentrationen über den im Test zu erfassenden Mindestmengen liegen. Soweit es sich um niedermolekulare Verbindungen handelt, ist ohnedies durch die vorausgehende Dialyse mit ihrer Eliminierung zu rechnen.

Da im Laufe der vielstündigen Perfusionen trotz Antibioticumzusatz mit dem Aufkommen von Bakterien zu rechnen war, die den Test durch $1-{ }^{14} \mathrm{C}$ Oxydation der Glukose verfälschen konnten, wurden Proben der Durchströmungsflüssigkeit bakteriologisch untersucht und dabei Keime der Alkaligenes-Gruppe außer Albumin neugebildeten Proteinanteil suchen dürfen.

LEONARDS ${ }^{30}$, GoLDBERG und EGDAHL ${ }^{18}$ und STEINKE et al. ${ }^{53}$ wiesen noch am 7 . und 8 . Tag im Plasma pankreatektomierter Tiere mit dem Fettgewebstest eine insulinartig wirkende Substanz nach, die sich nicht mit IAK hemmen ließ. SAMAAN et al. halten es für möglich, daß diese nach Pankreasexstirpation noch nachweisbare ILA durch Umwandlung von hemmbarem Insulin aus eventuell im Darm befindlichen Inselzellen durch die Leber entsteht ${ }^{44}$. Dies erscheint schon aus quantitativen Uberlegungen heraus wenig wahrscheinlich. Geht man davon aus, daß die nichthemmbare Serum-ILA beim pankreaslosen Tier um 50\% absinkt ${ }^{53}$, so müßte die Insulinproduktion des Darmes etwa der des Pankreas entsprechen. Wir möchten auf Grund unserer Untersuchungen eher annehmen, daß die nach Pankreatektomie persistierende, nichthemmbare Serum-ILA hepatischen Ursprungs und möglicherweise mit der hier beschriebenen hepatischen, nichthemm-

* Herrn Prof. Dr. G. Linzenmeier, Institut für Hygie. ne und Med. Mikrobiologie der Universität München, sind wir für die Durchführung dieser Untersuchungen zu groBem Dank verpflichtet. 
baren ILA identisch ist. In diesem Sinne wäre es auch zu verstehen, daß als Folge von Leberschädigungen, sei es dureh Anoxie im Anschluß an Ligierung der A. hepatica ${ }^{44}$, sei es durch Vergiftung mit Alloxan ${ }^{8,40}$ oder auch bei Leberkrankheiten ${ }^{42}$ der Serumspiegel an nichthemmbarer ILA regelmäßig absinkt.

Für großzügige Unterstützung der vorliegenden Arbeiten danken wir der Deutschen Forschungsgemeinschaft, Bad Godesberg, dem bayerischen Staatsministerium für Unterricht und Kultus und der Eli Lilly Com. pany, Indianapolis, USA.

Literatur. Beigeiman, P.M. and I.S. Oxoprienko: Insulin-like effects of serum albumin and globulin fractions on glucose uptake by rat epididymal adipose tissue. Diabetes 8, 438 - 441 (1959). - 2 Brauer, R.W., R.L. Pessotti and P. Ptzzonato: Isolated rat liver preparation. Bile production and other basic properties. Proc. Soc. exp. Biol. (N.Y.) 78, 174-181 (1951). -3 BRown, H., F. SANGER and R. KITAT: The structure of pig and sheep insulins. Biochem. J. 60, 556-565 (1955). - 4 BÜRGI, H., E.B. Ramsterer, E.R. Froesch, P. Bally und A. LabHaRt: ,Freies" und ,gebundones" Insulin im Serum von Patienten mit B-Inselzell-Adenom. Helv, med. Acta 29, 527-534 (1962). - ${ }^{5}$ Bürar, H., K. Kopetz, K. SchWARz and E.R. FroEsCH: Fate of rat insulin in rat-liver perfusion studied by adipose-tissue assay. Lancet $2,314-316$ (1963). - 6 CAHTLL, G.F., Jr., B. LebonuF and R.B. Furve: Studies on rat adipose tissue in vitro. VI. Effect of epinephrine on glucose metabolism. J. biol. Chem. 235, $1246-1250(1960) .-7$ CoHEN, S., A.H. Gordon and C. MatTHews: Catabolism of $\gamma$-globulin by the isolated perfused rat liver. Biochem. J. 82, $197-205$ (1962). $-{ }^{8} \mathrm{Coo}$ PERSTETN, S.J. and A. LAZAROW: Alloxan inactivation of coenzyme $A$ and the pigeon liver acetylating enzyme. $J$. biol. Chem. 232, 695-703 (1958). $-{ }^{9}$ Lit. vgl. Documenta Geigy, wiss. Tab., 6. Aufl. (1960), Seite 426. - ${ }^{10}$ Lit. vgl. Documenta Geigy, wiss. Tab. 6. Aufl. (1960), Seite 444. ${ }^{11}$ Lit. vgl. Documenta Geigy, wiss. Tab., 6. Aufl. (1960), Seite 446. - ${ }^{12}$ Lit. vgl. Documenta Geigy, wiss.Tab., 6. Aufl. (1960), Seite 447. - 13 Lit, vgl. Documenta Geigy, wiss. Tab., 6. Aufl. (1960), Seite 451. - 13a VALLEe, B.L. and J. G. GIBson: The zinc content of normal human whole blood, plasma, leucocytes, and erythrocytes. J. biol. chem. 176, 445-457 (1949). - 14 FAHEY, J. I., P.F. MCCOY and M. GoULIAN : Chromatography of serum proteins in normal and pathologic sera: the distribution of protein-bound carbohydrate and cholesterol, siderophilin, thyroxin-binding protein, $\mathbf{B}_{1}$-binding protein, alkaline and acid phosphatases, radioiodinated albumin and myeloma proteins. J. clin. Invest. 37, 272-284 (1958). - ${ }^{15}$ Freund, J., K. J. Тномson, H. B. HougH, H. E. Sommer and T.M. Pisani : Antibody formation and sensitization with the aid of adjuvants. J. Immunol. 60, 383-398 (1948). - 16 Froescr, E.R., H. Bürgi, E.B. Ramseier, P. Bally and A. LaBHART: Antibody-suppressible and nonsuppressible insulinlike activities in human serum and their physiologic sig. nificance. An insulin assay with adipose tissue of increased precision and specificity. J. clin. Invest. 42, 1816-1834 (1963). - ${ }^{17}$ Fromscr, E.R., H. Bürgr, W.A. MÜIJ.ER und A. LABHART : Mit Antiinsulin hemmbare und nicht-hemmbare Insulinaktivität im menschlichen Serum. Schweiz. med. Wsehr. 94, 309-313 (1964). — ${ }^{18}$ GOLDBERG, H.L. and R.H. FonaAL: Studies suggesting the extra-pancreatic production of substances with insulin-like activity. Fed. Proc. 20, 190 (1961). - ${ }^{19}$ GoRdon, A.H. and J.H. HUMPHREY : Methods for measuring rates of synthesis of albumin by the isolated perfused rat liver. Biochem. J. 75, 240-247 (1960). - ${ }^{20}$ Gondon, A.H.: Detection of liver proteins in circulating blood. Nature, Lond. 189, $727-728$ (1961). - 21 Grodsky, G.M. and P.H. Fors-
HAM: An immunochemical assay of total extractable insulin in man. J. clin. Invest. 39, 1070-1079, (1960). 22 HARRIS, J.T., F. SANGER and M.A. NAUGHTON: Species differences in insulin. Arch. Biochem. 65, 427-438 (1956). $-{ }^{23}$ HaUgaARD, N. and J.B. Marsh: Effect of insulin on the metabolism of adipose tissue from normal rats. J. biol. Chem. 194, 33-40 (1952). - ${ }^{24}$ Hořmsší, J. and R. Sine. TANA: The isolation of gamma globulin from blood-serum by rivanol. Acta med. scand. 155, 65-70 (1956). — 25 Isminara, Y., T. Sarto, Y. Itro and M. FuJtmo: Structure of sperm-and sei-whale insulins and their breakdown by whale pepsin. Nature, Lond. 181, $1468-1469$ (1958). ${ }^{26} \mathrm{KEEN}, \mathrm{H}$. : Properties of human "albumin". A metabolic study of albumin extracts from normal and diabetic plasma. Diabetes, 12, 406-413 (1963). - 27 KREBS, H.A. und K. Henserert: Untersuchungen über die Harnstoffbildung im Tierkörper. Hoppe-Seylers Z. physiol. Chem. 210, 33-66 (1932). ${ }^{28}$ LEBOEUF, B. and G.F. CAHILL, Jr.: Studies on rat adipose tissue in vitro. VIII. Effect of preparations of pituitary adrenocorticotropic and growth hormones on glucose metabolism. J. biol. Chem. 236, $41-46$ (1961). ${ }^{29}$ LEE, H.M., R.M. Elus and M. V. Sigar, Jr.: Some insulin-like effects of nicotinic acid observed with isolated rat epididymal adipose tissue. Biochim. biophys. Acta 49, 408-410 (1961). - ${ }^{30}$ LmONARDs, J.R.: Insulin-like activity of blood what it is ? Fed. Proc. 18, 272 (1959). - ${ }^{31}$ LI, C.H., N.R. MoudgaL and $H$. PAPKofr : Immunochemical investigations of human pituitary growth hormone. J. biol. Chem. 235, 1038-1042 (1960). - ${ }^{32}$ MASKE, H.: Beobachtungen über das Zink in den Langerhansschen Inseln des Pankreas und seine Beziehungen zur Inselfunktion. Z. Naturforsch. 8b, 96104 (1953). - ${ }^{33}$ Maske, H., H. Wolf'F und B. STAMPFL: Ửber die Verhinderung der diabetogenen Alloxanwirkung durch vorhergehende Glucosegaben. Klin. Wschr. 31, 79 -81 (1953). - ${ }^{34}$ Matschinsky, F., U. Meyer und. O. Wietand : Die Wirkung des Knollenblätterpilzgiftes Phalloidin auf die isolierte Rattenleber. Biochem. Z. 333, $48-61$ (1960). - ${ }^{35}$ Miller, L. L., C. G. BLY, M. L. WATson and W.F. BALE: Dominant role of liver in plasma protein synthesis; direct study of isolated perfused rat liver with aid of lysine- $\varepsilon-\mathrm{C}^{14}$. J. exp. Med. 94, 431-453 (1951). - ${ }^{36}$ Mrrsky, I.A. and G. Perisutit: Action of oxytocin and related peptides on epididymal adipose tissue of the rat. Endocrinology 71, 158-163 (1962). 37 Moloney, P.J. and M. Covas: Antigenicity of insulin: diabetes induced by specific antibodies. Biochem. J. 59, 179-185 (1955). -38 NICOL, D.S.H.W. and L.F. SMitTH: Amino-acid sequence of human insulin. Nature, Lond. 187, $483-485(1960)$. - 39 Pituman, J.A., B.R. Boshel, B.H. Wricrams, D. Hamner and P. Hrrr: Insulin-like activity of vasopressin and oxytocin. Biochem. biophys. Res. Comm. 6, 29-32 (1961). - 40 ResnIK, R.A. and A.R. Wouff: The reaction of alloxan with glutathione and protein. Arch. Biochom. 64, 33-50 (1956). il Samaax, N.A., W.J. Dempster, R. Fraser, N.W. Please and D. Stilliman: Further immunological studies on the form of circulating insulin. J. Endocrin. 24, 263277 (1962). - ${ }^{42}$ Samaan, N., D. Stillman and R. Fra SER: Abnormalities of serum insulin-like activity in liverdisease. Lancet 2, $1287-1290$ (1962). - ${ }^{13}$ SAmAAN, N.A., W.J. Dempster, R. Fraser and D. Stullman: Changes in levels of "atypical" circulating insulin after infusing "typical" insulin through the liver. J. Endocrin. 26, 1-10 (1963). - ${ }^{44}$ Samaan, N., R. Fraser and W.J. Dempster : The "typical" and ",atypical" forms of serum insulin. Diabetes 12, $339-348$ (1963). - ${ }^{45}$ Schimassek, H.: Metabolite des Kohlenhydratstoffwechsels der isoliert per. fundierten Rattenleber. Biochem. Z. 336, 460-467 (1963). - ${ }^{46}$ Siess, E.: Dissertation München 1965. - ${ }^{47}$ SIEss, E., A. Teinzer und O. Wrimand: Bildung nichthemmbarer „Insulin Like Activity“ (ILA) in der Rattenleber. Herbsttagung der Deutschen Gesellschaft f. physiol. Chemie Köln, 21. - 24. Okt. 1964. - ${ }^{48}$ Sress, E., A. TeINzER und 
O. WIELAND: Eine vereinfachte Fettgewebsmethode zur Insulinbestimmung im Serum. In Vorbereitung. - ${ }^{49} \mathrm{SIL}-$ VERMAN, J.L.: Glucosamine inhibition of $\left(1-\mathrm{C}^{\mathbf{1 4}}\right)$ glucose oxidation as measured by rat adipose tissue in vitro. Biochim. biophys. Acta 78, $94-100$ (1963). - ${ }^{50}$ SMIKIN, B. and $R$. ARce : Prolactin activity in blood during the normal human menstrual cycle. Proc. Soc. exp. Biol. N.Y. 113 485-488, (1963). - ${ }^{51}$ Slatme, J.D.H., N. SAMAan, R. Fraser and D. StmLiman: Immunological studies with circulating insulin. Brit. med. J. 1, 1712-1715 (1961). ${ }^{52}$ Soler, H.A., F.J. GuTter, M.M. WyCkOFF and E. A. Peterson : Chromatography of proteins. II. Fraetionation of serum protein on anion-exchange cellulose. J. Amer. chem. Soc. 78, 756-763 (1956). - Б3 Sternke, J., A. SiReK, V. Lauris, F.D.W. Lukens and A.E. Renold: Measurement of small quantities of insulin-like activity with rat adipose tissue. III. Persistence of serum insulin like activity after pancreatectomy. J. clin. Invest. 41, 1699-1707 (1962). - 54 Unger, R.H., A.M. EIsENTRAUT and L.L. MaDIson: The effects of total starvation upon the levels of circulating glucagon and insulin in man. J. clin. Invest. 42, $1031-1039$ (1963). - ${ }^{55} \mathrm{WEIG}_{\mathrm{EI}}$ LE, W.O., F.J. Dixon and M.P. Deichimileer: Effect of mycobacteria on antibody production induced by water- in-oil emulsions of soluble protein antigens. Proc. Soc, exp. Biol. N. Y. 105, 535-541 (1960). -56 WILSON, S. and $G$.H. Dixon : A comparison of cod and bovine insulins. Nature, Lond. 191, 876-879 (1961). — 57 WrNEGRAD. A.I., W. N. ShaW, F.D.W Lukens, W.C. Stadie and A.E. RENOLD: Effects of growth hormone in vitro on the metabolism of glucose in rat adipose tissue. J. biol. Chem. 234, 1922-1928 (1959). - 58 WINEGRAD, A.I., W.N. SHaW, F.D.W. Lukens and W.C. Stadie: Effects of prolactin in vitro on fatty acid synthesis in rat adipose tissue. J biol. Chem. 234, 3111-3114 (1959). - ${ }^{59}$ Wör NER, H. und K.F. Weinges: Über den Einfluß von Insulin und Glukagon auf den Glucosestoffwechsel des epididymalen Fettanhanges der Ratte in vitro. II. Der Einfluß von Glukagon im Vergleich mit dem Insulineffekt. Klin. Wschr. 39, 243--246 (1961). - 60 YAGI, Y., P. Mamer and D. Pressman: Two different anti-insulin antibodies in guinea pig antisera. J. Immunol. 89, 442-451 (1962).

Prof. Dr. O. WIELAND, Laboratorium für Klinische Chemie u. Biochemie der II. Medizinischen Universitätsklinik 8, München 